\title{
A Secure Online Based Friend Recommendation System for Social Network
}

\author{
Priyanka Uchekar ${ }^{1}$, Kirtee Gawade ${ }^{1}$, Arati Dhumal ${ }^{1}$, Rina Sandbhor ${ }^{1}$, Prof.S.D Kadam ${ }^{1}$, Prof. P.B.Sahane ${ }^{1}$ \\ Computer Engineering Department, P.K.Technical Campus Chakan, Maharashtra, India ${ }^{1}$
}

\begin{abstract}
In this paper, we have presented aThe modern Activity based friend recommendationservices. Social networking sites imply friend recommendation Systems in contribution to providing better user experiences. Online friend recommendation is a rapid developing topic in web mining. Current social networking servicing recommend friends to users based on their social graphs and mutual friends, which may not be the most appropriate to reflect a user's taste on friend selection in real lifetime. In this paper propose a system that recommends friends based on the daily activities of users. Here a semantic based friend recommendation is done based on the users' life styles. By using text mining, we display a user's everyday life as life archives, from which his/her ways of life are separated by using the Latent Dirichlet Allocation algorithm. At that point we discover a similarity metric to quantify the similarity of life styles between users, and as certain users' effect as far as ways of life with a similarity matching diagram. At last, we incorporate a feedback component to further enhance the proposal precision.
\end{abstract}

Keywords: ActivityRecognition;Social Network, Data Mining ;Pattern Recognition;Secerte Sharing Scheme.

\section{INTRODUCTION}

In your everyday lifestyles, organic meats have a huge will prove to be largest domain in understanding the user selection of pursuits, which in turn kind important behavior. One of the best examples of social networking is sequences of which shape our lifestyles. With this paper, FACEBOOK. According to current news Facebook is we all utilize phrase exercise to particularly consider the trying to develop algorithm, to understand user behavior. actions taken in this order connected with seconds, for Social Networking sites can help us in getting important example "sitting", "walking", or "typing", even though information of users, such as age, gender, location, weall utilize term way of living to consider higher-level language, actives, likes etc. our model takes into account abstractions connected with everyday lifestyles, for these parameters of the user to recommend books. Most of example "office work"of"shopping". In particular, this the friend suggestions mechanism relies on pre-existing "shopping" way of living mostly consists of this user relationships to pick friend candidates. For example, "walking" exercise, however might also secure Facebook relies on asocial link analysis among those who the"standing" or this "sitting" pursuits. To style everyday already share common friends and lifestyles adequately, we all bring an analogy in between recommendssymmetricalusersaspotential friends. The people's everyday lifestyles along with papers.Existing rules to group people together include:

social networking services recommend friends to users 1) Habits or life style

based on their social graphs, which may not be the most 2) Attitudes

appropriate to reflect a user's preferences on friend 3) Tastes

selection in real life. Prompted through this particular, 4) Moral Standards

likewise, we can address our everyday lifestyles (or 5) Economic level; and

lifestyle documents) seeing that a number of standards of 6) People they already know.

living (or topics), along with every single way of living Apparently, rule \#3 and rule \#6 are the mainstream factors seeing that a number of pursuits (or words).Monitor herein Considered by existing recommendation systems.

essence, we all signify everyday lifestyles using "life documents", as their semantic explanations are generally shown by way of their matters, which are standards of living in your research. Much like terms work for the reason that time frame connected with papers, people's pursuits normally work for the reason that Primitive vocab of these lifestyle papers.Social networking sites are used intensively from last decade. According to the current survey, Social Networking sites have the largest data set of users. Each social networking site notes/records each and every activity of user (like: what user likes? what user is doing? what is user's hobby? Etc.).SocialNetworking site

\section{RELATED WORK}

In [2] authors used average residual battery level of the entire network and it was calculated by adding two fields to the RREQ packet header of a on-demand routing algorithm i) average residual battery energy of the nodes on the path ii) number of hops that the RREQ packet has passed through.According to their equation retransmission time is proportional to residual battery energy. Those nodes having more battery energy than the average energy will be selected because its retransmission time will be 
less. Small hop count is selected at the stage when most of the nodes have same retransmission time. Individual battery power of a node is considered as a metric to prolong the network lifetime in [3]. Authors used an optimization function which considers nature of the packet, size of the packet and distance between the nodes, number of hops and transmission time are also considered for optimization. In [ 4] initial population for Genetic Algorithm has been computed from the multicast group which has a set of paths from source to destination and the calculated lifetime of each path. Lifetime of the path is used as a fitness function. Fitness function will select the highest chromosomes which is having highest lifetime. Cross over and mutation operators are used to enhance the selection. In [5] authors improved AODV protocol by implementing a balanced energy consumption idea into route discovery process. RREQ message will be forwarded when the nodes have sufficient amount of energy to transmit the message otherwise message will be dropped. This condition will be checked with threshold value which is dynamically changing. It allows a node with over used battery to refuse to route the traffic in order to prolong the network life. In [6] Authors had modified the route table of AODV adding power factor field. Only active nodes can take part in rout selection and remaining nodes can be idle. The lifetime of a node is calculated and transmitted along with Hello packets. In [7] authors considered the individual battery power of the node and number of hops, as the large number of hops will help in reducing the range of the transmission power. Route discovery has been done in the same way as being done in on-demand routing algorithms. After packet has been reached to the destination, destination will wait for time $\delta \mathrm{t}$ and collects all the packets. After time $\delta \mathrm{t}$ it calls the optimization function to select the path and send RREP. Optimization function uses the individual node's battery energy; if node is having low energy level then optimization function will not use that node.

\section{III.PROPOSED ALGORITHM}

\section{Fp-Growth Algorithm:}

Mine frequent itemsets using an FP-tree by pattern fragment growth.

Input:

- D, a transaction database;

- min sup, the minimum support count threshold.

Output:

- $\quad$ The complete set of frequent patterns.

\section{Method:}

1.The FP-tree is constructed in the following steps:

(a) Scan the transaction database D once.Collect F,the set of frequent items, and their support counts.sort $\mathrm{F}$ in support count descending order as L, the list of frequent items. (b) Create the root of an FP-tree, and label it as "null".For each transactionTrans in D do the following. Select and sort the frequent items in Trans according to the order of L.Let the sorted frequent item list in Trans be $[\mathrm{p}-\mathrm{P}]$, where

$\mathrm{p}$ is the first element and $\mathrm{P}$ is the remaining list.Call insert tree $([\mathrm{p}-\mathrm{P}], \mathrm{T})$, which is performed as follows.If $\mathrm{T}$ has a child $\mathrm{N}$ such that N.item-name=p.itemname, then increment N's count by 1 ; else create a new node $\mathrm{N}$, and let its count

be 1 , its parant link be linked to $\mathrm{T}$, and its node-link to the nodes with the same item-name via the node-link via the node-link structure.If $\mathrm{P}$ is nonempty, call insert tree $(\mathrm{P}, \mathrm{N})$ recursively.

1. The FP-tree is mined by calling FP growth(FP tree, null), which is implementedas follows.

procedure FP-growth(Tree, alpha) 1.if Tree contains a single path $\mathrm{P}$ then

2. for each combination(denoted as beta) of the nodes in the path $\mathrm{P}$

3. generate pattern beta union alpha with support count=minimun support countof nodes in beta;

4. else for each in the header of Tree $f$

5. generate pattern beta=aiU alpha with support count=ai.support count;

6. .construct beta's conditional pattern base and the beta's and then beta's conditionalFP tree Tree beta;

7. call FP-growth(Tree beta, beta); \}

\section{AES Algorithm:}

AES is short for Advanced Encryption Standard. AES is a symmetric encryption algorithm processing data in block of 128 bits. AES is symmetric since the same key is used for encryption and the reverse transformation, decryption. The only secret necessary to keep for security is the key. AES may configured to use different key-lengths, the standard defines 3 lengths and the resulting algorithms are named AES-128, AES-192 and AES-256 respectively to indicate the

length in bits of the This standard explicitly defines the allowed values for the key length $(\mathrm{Nk})$, block size $(\mathrm{Nb})$, and number of rounds $(\mathrm{Nr})$.

\section{Steps in AES Encryption:}

- Sub Bytesa non-linear substitution step where each byte is replaced with another according to a lookup table.

- Shift Rowsa transposition step where each row of the state is shifted cyclically a certain number of steps.

- Mix Columnsa mixing operation which operates on the columns of the state, combining the four bytes in each column.

- Add Round Keyeach byte of the state is combined with the round key; each round key is derived from the cipher key using a key schedule 


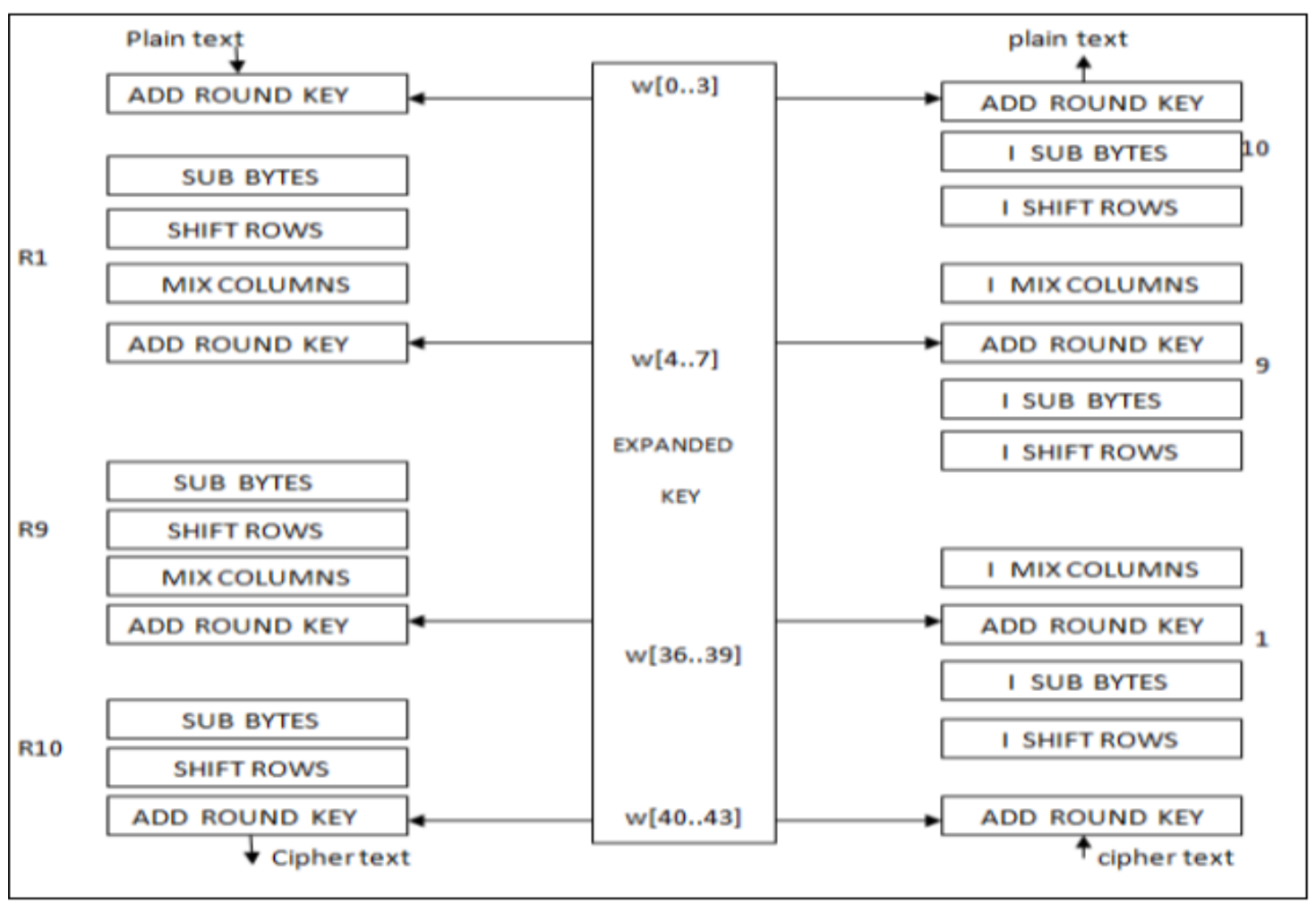

Figure A.1: General structure of AES algorithm.

\section{IV.FRAMEWORK OFPROPOSE SYSTEM}

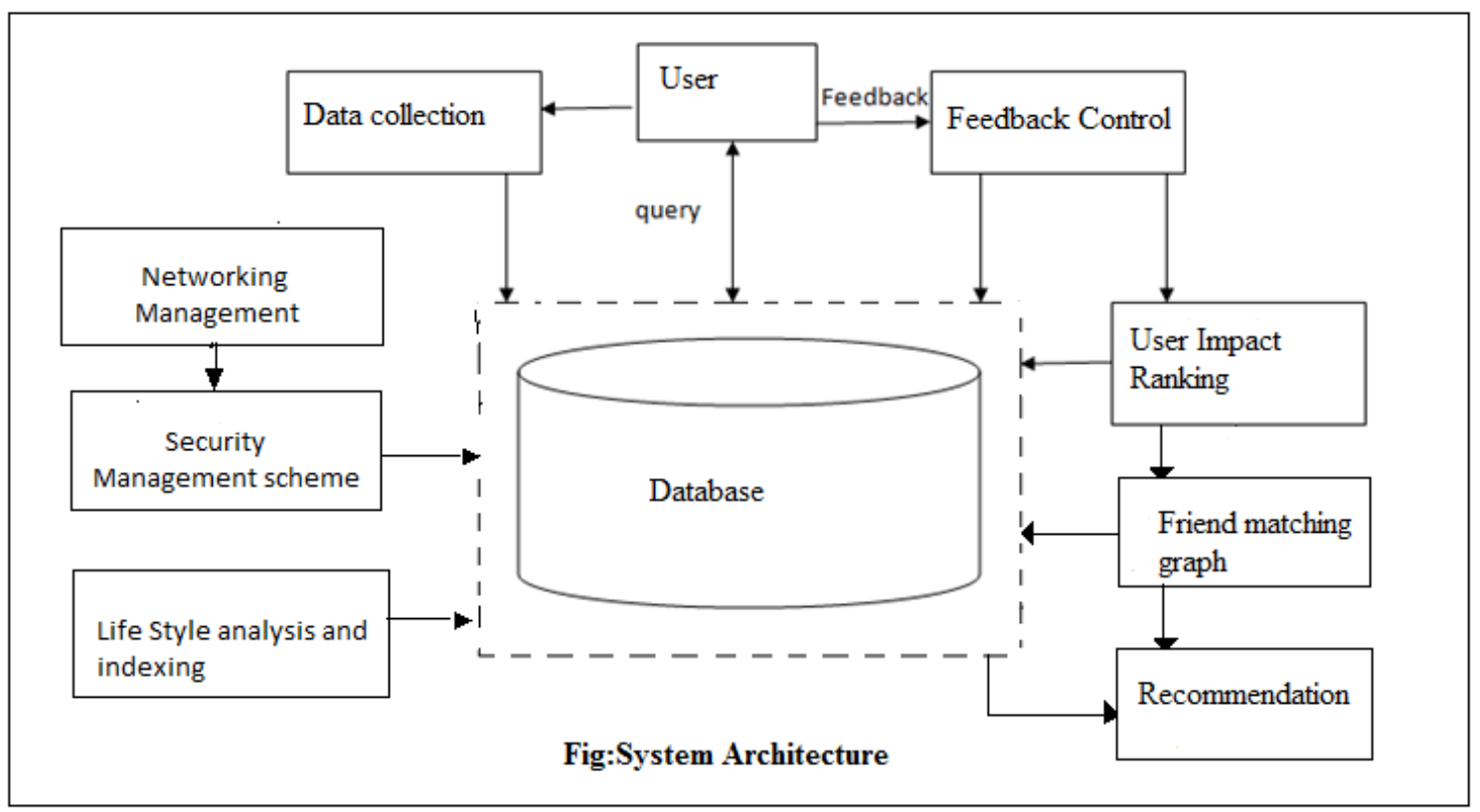

Figure. Framework of propose System

1.User:

name of user, delete the friend request.submit the In this framework user is initial acter.It is doing the all information and save the information. activity on network as like create account, update photo, search friend, send request to friend, accept friend request, 2.Data collection:

like photo, like comments.It is fire the query as like enter In this data collection box consist of user data collected.It the email id, mobile no.Update mobile no, update the collect the information to data update the dynamic data. 
3.Life style analysis and indexing:

It is store the user information like behaviour of user its like, dislike, interest, etc.Its all information analysis and indexing of users.

\section{Friend matching graph:}

It is store the information and drawing the friend matching graph as like one friend interset on singing song and other also then draw the friend matching graph.

\section{User impact ranking:}

It is give the impact of user by using threshold count.suppose threshold count is 4 then friend threshold count 4,5,6,7,3 then the ranking of friend recommendation as like $7,5,4,3$.
6.Recommendation:

It is show the friend recommendation list.Its recommend the friend on intrest and same mentality.

\section{Database:}

Database store the all information of user perform in network update the database dynamically.

8.Security management:

All the facebook database provide the security using AES and DES algorithm. Its provide security like plaintext to hypertext or supertext data provide the encryption and decryption.

9.User feedback:

It is store the user feedback as like its benifical or not.

\section{SIMULATION RESULTS}

1.User login page:-
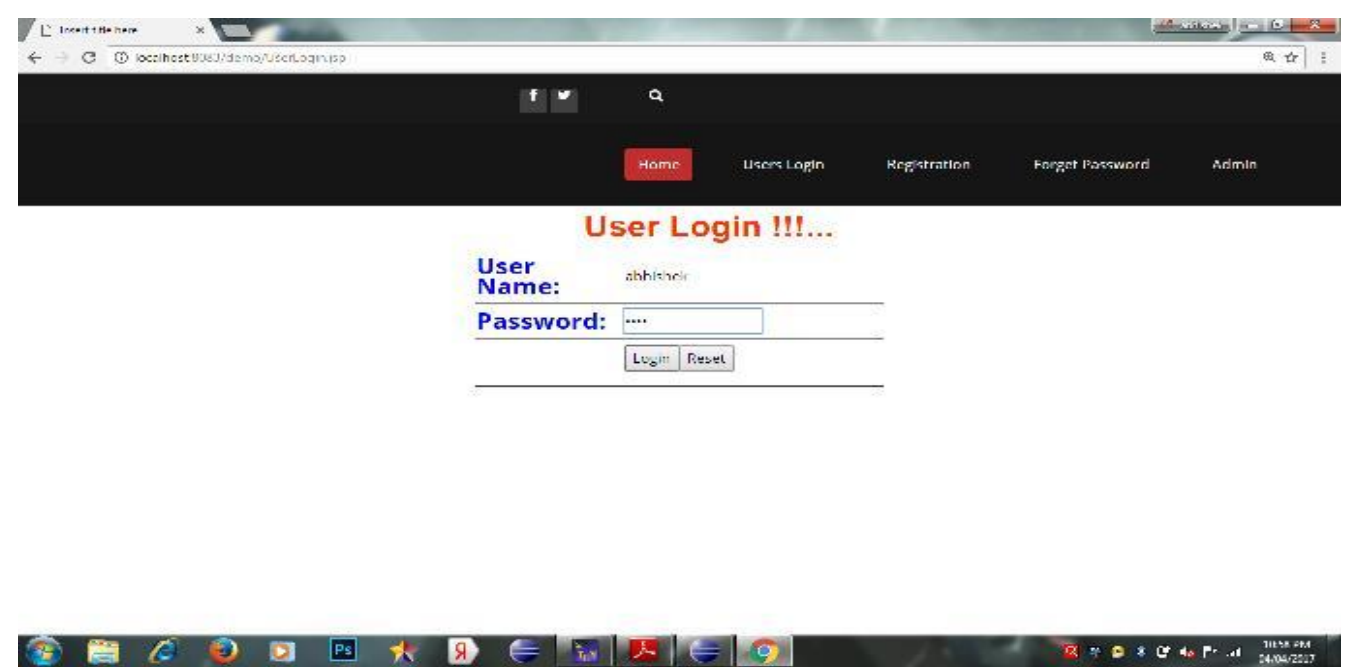

2.Admin login page:-

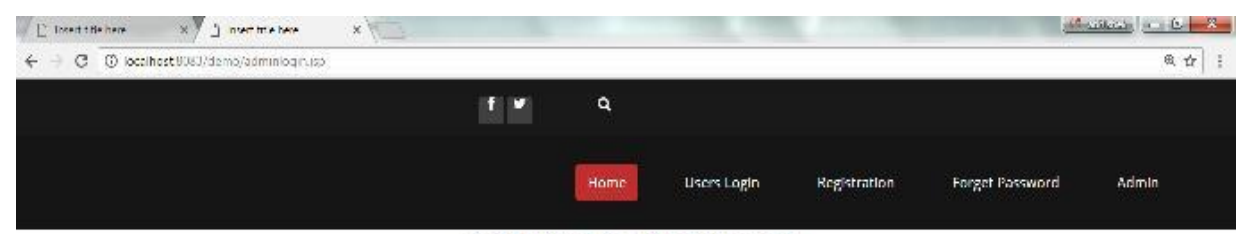

Admin Login Form !

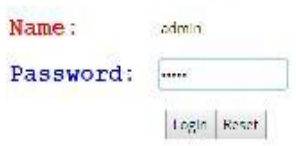


International Journal of Advanced Research in Computer and Communication Engineering ISO 3297:2007 Certified

Vol. 6, Issue 3, March 2017

3.Users ranking depends on similairty matching:-

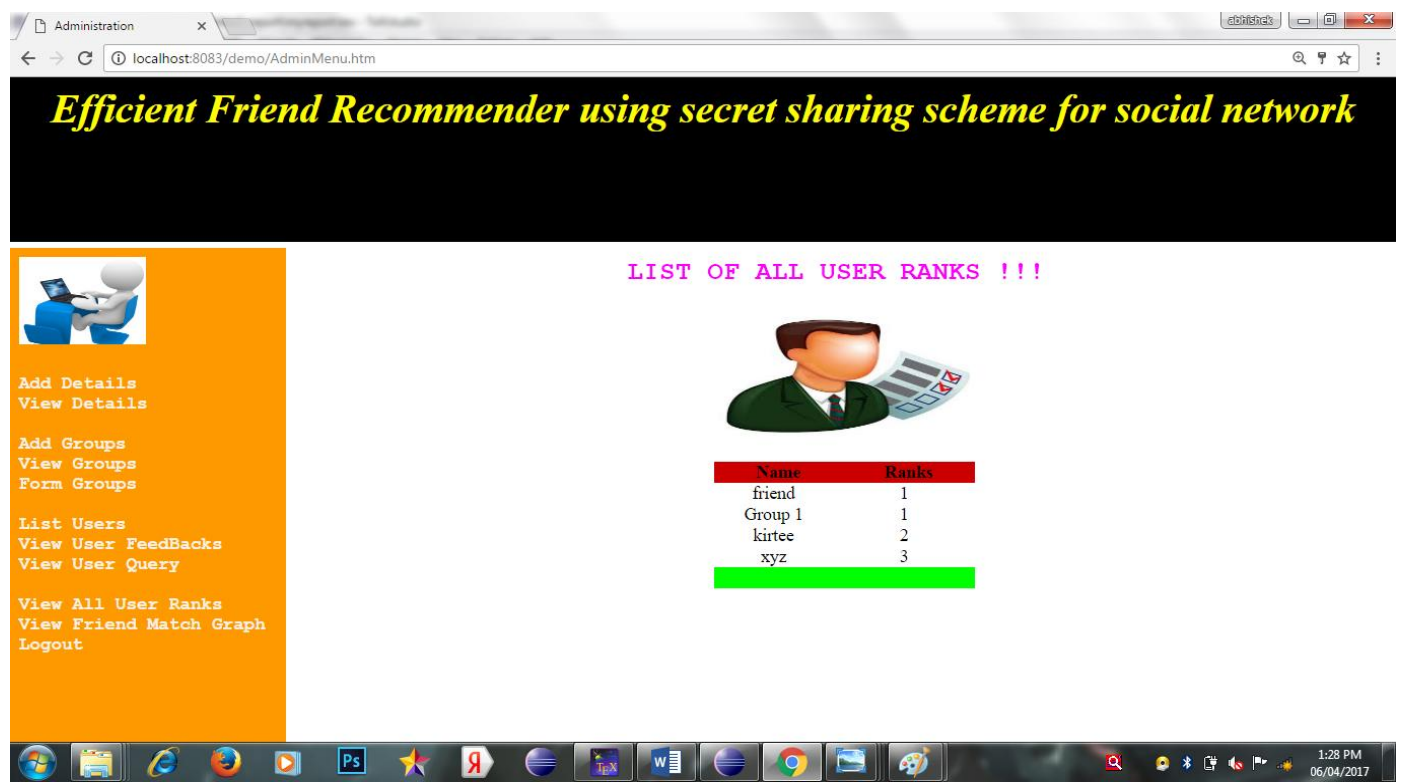

4.Friend matching graph:-

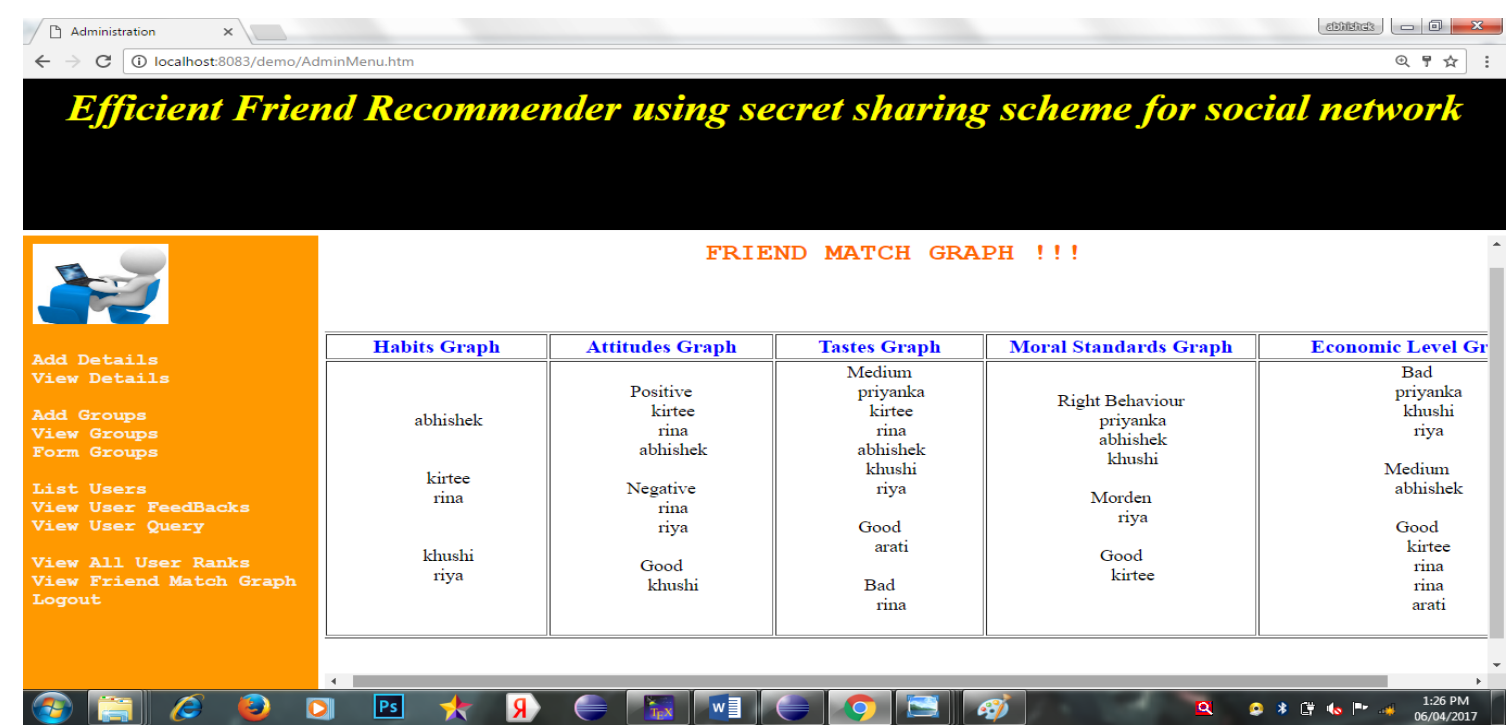

5.Recommendation:-

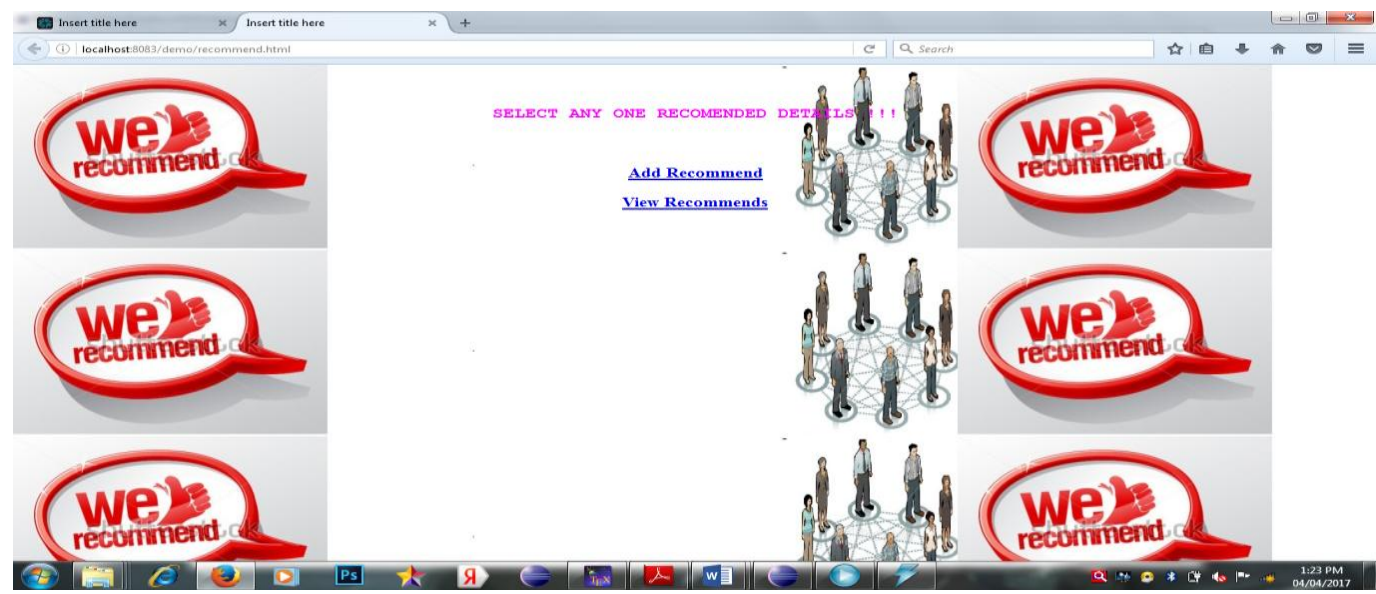


6.Add Recommendation :-

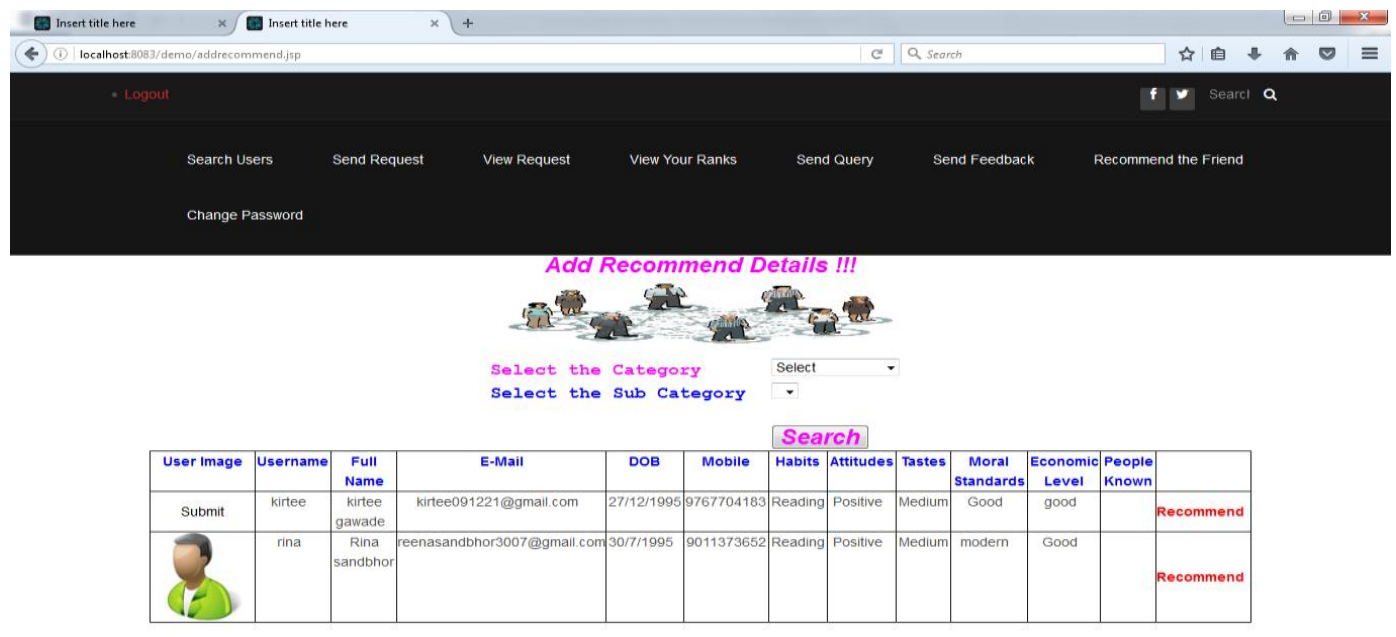

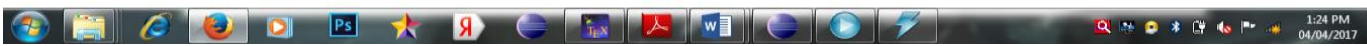

7.View recommendation:-
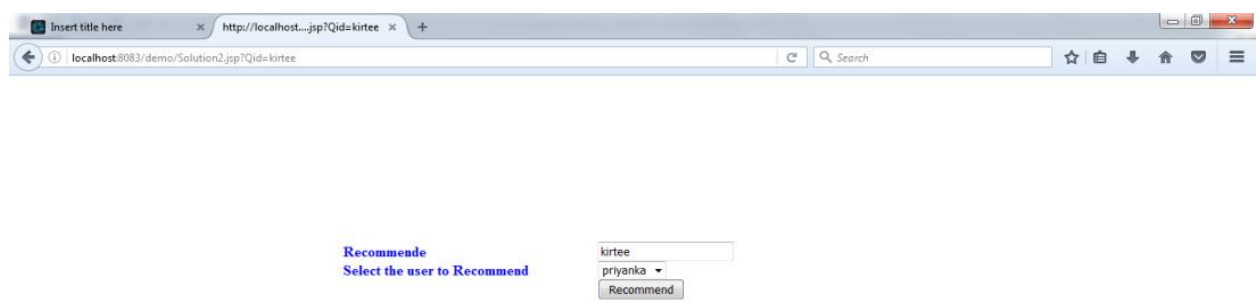

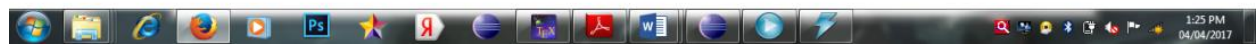

8.Mail for security and password purpose:-

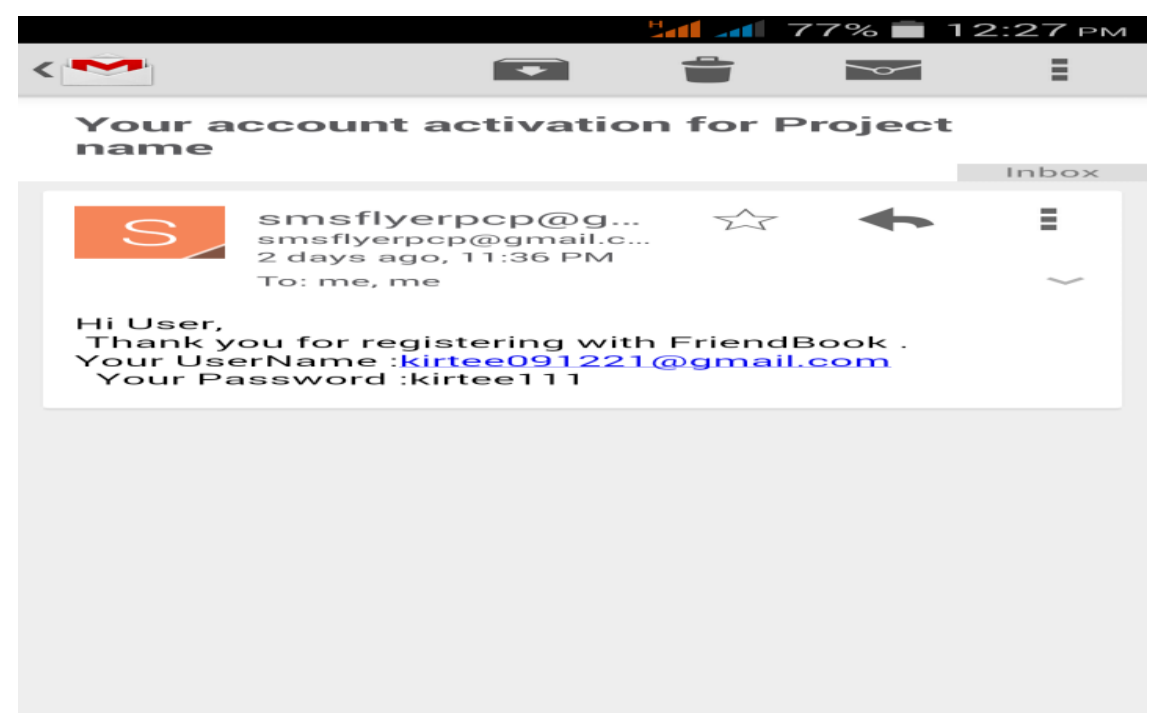




\section{VI.CONCLUSION AND FUTURE WORK}

In this project, we designed and implemented the Friendbook, a semantic-based friend recommendation system for social networks. Is is Different from the other friend recommendation mechanisms relying on social graphs in existing social networking services, where as this Friendbook extracted life styles from user-centric data collected from sensors on the Smartphone and recommended potential friends to users if they share similar life styles.

We implemented Friendbook on the Android-based Smartphone's, and evaluated its performance on both small scale experiments and large-scale simulations. The results showed that the recommendations accurately reflect the preferences of users in choosing friends. Beyond the current prototype, the future work can be fourfold. First, we would like to evaluate our system on largescale field experiments. Second, we intend to implement the life style extraction using LDA and the iterative matrix-vector multiplication method in user impact ranking incrementally, so that Friendbook would be scalable to large-scale systems.

Third, the similarity threshold used for the friendmatching graph is fixed in our current prototype of Friendbook. It would be interesting to explore the adaption of the threshold for each edge and see whether it can better represent the similarity relationship on the friend-matching graph. At last, we plan to incorporate more sensors on the mobile phones into the system and also utilize the information from wearable equipments (e.g., Fitbit, iwatch, Google glass, Nike+, and Galaxy Gear) to discover more interesting and meaningful life styles).

\section{REFERENCES}

1. Zhibo Wang, Qing Cao, and Zhi Wang, "Friend-book: A Semanticbased Friend Recommendation System For Social Networks", IEEE TRANSACTIONS ON MOBILE COMPUTING."

2. Facebook statistics. http://www.digitalbuzzblog.com/ facebookstatistics- stats-facts-2011/.

3. L.Bian and H.Holtzman. "Online friend recommendation throughpersonality matching and collaborative filtering". Proc. Of UBICOMM, pages 230-235, 2011.

4. J. Kwon and S. Kim." Friend recommendation method using physical and social context ". International Journal of Computer Science and Network Security, 10(11):116-120, 2010.

5. X. Yu, A Pan, L.-A. Tang, Z. Li, and J. Han. "Geo-friends recommendation in gaps-based cyber-physical Social network".Proc. of ASONAM, pages 361-368, 2011.

6. W. H. Hsu, A. King, M. Paradesi, T. Pydimarri, and T. Weninger. Collaborative and structuralrecommendation of friends using weblog-based social network analysis. Proc. of AAAI SpringSymposium Series, 2006.

7. S-T. Kuan, B.FY. Wu and W.FJ. Lee, "Finding friend groups in blogosphere," in Advanced Information Networking and Applications; Workshops, 2008. AINAW 2008. 22nd International Conference on, mar.2008, pp. $1046-1050$

8. P. Desikan, N. Pathak, and J. Srivastava, V. Kumar, "Incremental page rank computation on evolving graphs," in Proc. Special Interest Tracks Posters 14th Int. Conf. World Wide Web, 2005, pp.1094-1095.
9. N. Eagle and A. S. Pentland, "Reality mining: Sensing complexsocial systems," Pers. Ubiquitous Computer, vol. 10, no. 4, pp.255-268, Mar. 2006.

10. K. Farrahi and D. Gatica-Perez, "Probabilistic mining ofsociogeographicroutines from mobile phone data," IEEE J. Select.Topics Signal Process, vol. 4, no. 4, pp. 746-755, Aug. 2010.

11. K. Farrahi and D. Gatica Perez, "Discovering routines from largescale human locations using probabilistic topic models,"ACMTrans. Intel. Syst. Technol., vol. 2, no. 1, pp. 3:13:27, 2011.

12. B. A. Frigyik, A. Kapila, and M. R. Gupta, "Introduction to theDirichlet distribution and related processes," Dept. Elect.Eng.,Univ. Washington, Seattle, WA, USA, UWEETR-20100006,2010 .

13. A. Giddens, Modernity and Self-Identity: Self and Society in theLate Modern Age. Stanford, CA, USA: Stanford Univ. Press, 1991.

14. L. Gou, F. You, J. Guo, L. Wu, and X. L. Zhang, "Sfviz: Interestbased friends exploration and recommendation in social networks,"in Proc. Visual Inform. Commun.-Int. Symp., 2011, p. 15

15. W. H. Hsu, A. King, M. Paradesi, T. Pydimarri, and T. Weninger,"Collaborative and structural recommendation of friends usingweblog-based social network analysis," in Proc. AAAI Spring Symp. Ser., 2006, pp. 55-60.

16. T. Huynh, M. Fritz, and B. Schiel, "Discovery of activity patternsusing topic models," in Proc. 10th Int. Conf. Ubiquitous Comput.,2008, pp. 10-19.

17. J. Kwon and S. Kim, "Friend recommendation method usingphysical and social context," Int. J. Comput. Sci. Netw. Security,vol. 10, no. 11, pp. 116-120, 2010. 